Article

\title{
Charge Transport in LDPE Nanocomposites Part II-Computational Approach
}

\author{
Anh T. Hoang, Yuriy V. Serdyuk and Stanislaw M. Gubanski * \\ Division of High Voltage Engineering, Department of Materials and Manufacturing Technology, \\ Chalmers University of Technology, Gothenburg SE-41296, Sweden; anh.hoang@chalmers.se (A.T.H.); \\ yuriy.serdyuk@chalmers.se (Y.V.S.) \\ * Correspondence: stanislaw.gubanski@chalmers.se; Tel.: +46-31-772-1616
}

Academic Editor: Frank Wiesbrock

Received: 28 January 2016; Accepted: 16 March 2016; Published: 23 March 2016

\begin{abstract}
A bipolar charge transport model is employed to investigate the remarkable reduction in dc conductivity of low-density polyethylene (LDPE) based material filled with uncoated nanofillers (reported in the first part of this work). The effect of temperature on charge transport is considered and the model outcomes are compared with measured conduction currents. The simulations reveal that the contribution of charge carrier recombination to the total transport process becomes more significant at elevated temperatures. Among the effects caused by the presence of nanoparticles, a reduced charge injection at electrodes has been found as the most essential one. Possible mechanisms for charge injection at different temperatures are therefore discussed.
\end{abstract}

Keywords: low-density polyethylene; nanocomposites; charge transport; dc conductivity; charge injection; trapping; de-trapping; charge carrier mobility

\section{Introduction}

Accumulation of space charges in polymeric insulation is the main concern during the operation of high voltage direct current (HVDC) cables. It may greatly enhance the electric field inside the insulation bulk that eventually has a detrimental effect on the life expectancy of cables. Thus, knowledge of the generation and transport of charge carriers plays an important role in designing reliable insulation systems of HVDC cable working at high electric field strength. Nowadays, various experimental techniques are used to assess charge dynamics in insulating materials. Apart from that, computer simulations have become popular since they offer great flexibility in investigating effects of different factors and in conducting parametric studies.

A pioneering computer model considering transient processes of charge generation and transport in cable insulation exposed to dc stresses was published in 1994 by Alison and Hill [1] with the aim of reproducing space charge accumulation attained experimentally [2] on a $2.5 \mathrm{~mm}$ thick sample of cross-linked polyethylene (XLPE). The model incorporated charge generation due to injection at insulation-electrode interfaces and its transport through material bulk associated with trapping and recombination. Since that time, a variety of models [3-11] were developed for studying different physical processes taking place in polyethylene (PE) under a high dc electric field. Le Roy et al. [6] proposed a model accounting for de-trapping of charges from deep trapping sites (in contrast to earlier works $[1,3,4])$ that yielded consistent prediction of experimental results on space charge distribution, conduction currents, and electroluminescence in low-density polyethylene (LDPE). Furthermore, Boufayed et al. [7] introduced more realistic exponential distribution of traps instead of two single trap levels (shallow and deep traps) utilized in other models $[1,3,5,6]$. Most recently, a contribution of surface states at the interfaces between dielectric and electrodes to the dynamics of space charges in 
LDPE films has been considered [10]. Additionally, formation of charge packets in PE arising as the applied electric field exceeds $100 \mathrm{kV} / \mathrm{mm}$ has been studied in $[4,11]$.

It is worth noting that most of the reported simulations were performed for ambient temperatures $\sim 20{ }^{\circ} \mathrm{C}$, which is not the working condition of cable insulation in reality. The actual operating temperatures may reach $\sim 70-80{ }^{\circ} \mathrm{C}$. Moreover, a temperature gradient across the insulation may exist, which affects local characteristics of the material relevant to charge transport. These facts raise questions on the applicability of the existing models for predicting the behavior of insulation in practical situations. This difficulty, in fact, has been dealt with in [9], where the distribution of the electric field and space charges in a cable working under isothermal and temperature gradient conditions were modelled by assuming dependencies of charge carriers' mobility on temperature and electric field while setting all other model parameters the same as in [6]. The simulated results, however, have not been confirmed by respective experimental data yet.

As for simulations of charge transport in nanocomposites, information about such studies is rather limited. To formulate a consistent model for this case, the basic model of charge transport in pure polymers needs to be extended to account for formation of traps associated with nanofiller particles. These trapping sites may stimulate specific processes which are not present in pure materials. In particular, formation of deep traps that capture mobile carriers injected from electrodes, thus preventing further generation of charges at electrode-material interfaces has been introduced in [12]. Computer simulations utilizing parametric studies to examine the hypotheses as well as to compare contributions of different processes have been reported in $[13,14]$. The general requirement to such models is that they should be capable to explain the facts that the addition of nanofillers into polymers leads to greatly reduced material dc conductivity [15-17] and significantly limited accumulation of space charges $[16,18,19]$ (that actually make, e.g., PE nanocomposites favorable materials for HVDC cable insulation). As for today, such a consistent model of charge transport in nanocomposites that can reproduce experimental results is still lacking and, hence, it needs to be developed.

In the present paper, we study charge transport in LDPE with and without nanofillers at different temperatures by computer simulations using COMSOL Multiphysics (COMSOL AB: Stockholm, Sweden). As a number of processes, i.e., charge generation and transport as well as charge trapping, de-trapping, and recombination have to be accounted for, numerous parameters are involved in the model. It is a common practice to derive these parameters by using fitting procedures that result in additional uncertainties in the model. For avoiding this, we use values of parameters, in particular, the mobility of charges derived from experimental data presented in the first part of this work [17]. The validation of the developed model is examined by comparing the simulated results with conduction currents measured for unfilled LDPE and LDPE $/ \mathrm{Al}_{2} \mathrm{O}_{3}$ nanocomposite.

\section{Model of Charge Transport in Insulating Polymers}

\subsection{The Model}

Following the experimental conditions of [17], we consider a flat sample of insulating material of thickness $L$ that is sandwiched between a semiconducting anode and a stainless steel cathode. A positive dc voltage $V_{0}$ is applied to the anode at zero time, while the cathode is grounded. Since the radii of the electrodes are much larger than the thickness of material sample, the edge effect can be neglected. Thus, the study of charge transport in the flat sample can be reduced to a one-dimensional domain. In such a case, most of the parameters described below are functions of coordinate $x$ along insulation thickness and time $t$ (note that these dependencies are usually omitted in mathematical expressions below).

To describe conduction process under given conditions, bipolar charge transport model $[1,6]$ is employed. In the model, charge carriers in the material appear due to injection of holes at the anode and electrons at the cathode. The injected charge carriers drift through the material bulk due to the electric field and their transfer is affected by two types of localized states, namely, shallow and deep 
traps. The former trap type is attributed to structural defects in materials, such as folds, kinks, or ends of polymeric chains. The latter trap type has chemical origin due to the presence of reactive groups such as carbonyl $(-\mathrm{C}=\mathrm{O})$, carboxyl $(-\mathrm{COOH})$, etc. Charge carriers transported between shallow traps (by hopping) are referred as mobile electrons and holes, whereas the ones being captured in the deep trapping centers are referred as trapped electrons and holes. The trapped carriers occupy traps for a certain residence time, which is considerably longer than that the mobile carriers spend in shallow traps. Charged species captured in deep traps can be released back to the transport state through a de-trapping process. Furthermore, the drift of charge carriers through the material is also associated with their irreversible losses due to various types of recombination. The mathematical description of the model is provided below. Note that model parameters and other quantities in the following equations related to mobile electrons and holes and trapped electrons and holes are denoted by subscripts $e$ and $h$, etr and $h t r$, respectively.

As mentioned, charge carriers can be injected into the insulation through both electrodes as a high dc electrical field is applied. By assuming Schottky's mechanism, the densities of injected currents can be expressed as:

$$
\begin{aligned}
& j_{e}(0, t)=A T^{2} \exp \left(-\frac{q\left(\phi_{K}-\Delta \phi_{K}\right)}{k T}\right) \\
& j_{h}(L, t)=A T^{2} \exp \left(-\frac{q\left(\phi_{A}-\Delta \phi_{A}\right)}{k T}\right)
\end{aligned}
$$

Here, the coordinates of the cathode and anode are respectively 0 and $L, \mathrm{~m} ; A$ is Richardson's constant $\left(A=1.2 \times 10^{6} \mathrm{~A} \cdot \mathrm{m}^{-2} \cdot \mathrm{K}^{-2}\right)$; $T$ being absolute temperature, $\mathrm{K} ; q$ is elementary charge $\left(q=1.6 \times 10^{-19} \mathrm{C}\right)$; $\phi_{\mathrm{A}}$ and $\phi_{\mathrm{K}}$ are respectively the barrier heights for charge injection from the anode and cathode, eV; $k$ stands for Boltzmann's constant $\left(k=1.38 \times 10^{-23} \mathrm{~J} \cdot \mathrm{K}^{-1}\right)$. In Equations (1) and (2), $\Delta \phi_{\mathrm{A}, \mathrm{K}}$ denote the field-lowered barrier heights for charge injection due to electric field $E_{\mathrm{A}, \mathrm{K}}$ at corresponding electrodes:

$$
\Delta \phi_{A, K}=\sqrt{\frac{q E_{A, K}}{4 \pi \varepsilon_{0} \varepsilon_{r}}}
$$

where $\varepsilon_{0}=8.854 \times 10^{-12} \mathrm{~F} \cdot \mathrm{m}^{-1}$ is the permittivity of vacuum and $\varepsilon_{r}$ being material relative permittivity.

Transport of injected charge carriers through insulating materials is governed by a system constituting transport equation (4), current continuity equation (5), differential equation (6), and Poisson's equation (7):

$$
\begin{gathered}
j_{e, h}(x, t)=q \mu_{e, h} n_{e, h}(x, t) E(x, t) \\
\frac{\partial n_{e, h}(x, t)}{\partial t}+\frac{1}{q} \frac{\partial}{\partial x}\left(j_{e, h}(x, t)\right)=S_{e, h}(x, t) \\
\frac{\mathrm{d} n_{e t r, h t r}(x, t)}{\mathrm{d} t}=S_{e t r, h t r}(x, t) \\
\nabla\left(\varepsilon_{0} \varepsilon_{r} E(x, t)\right)=\rho(x, t)
\end{gathered}
$$

Note that in the transport equation (4) only the drift current is presented, whereas the diffusion current caused by a non-zero gradient of charge densities is neglected. The contribution of diffusion to conduction process has been revealed to be insignificant through additional simulations performed in models with and without considering it. For sake of clarity, the results obtained in these calculations are not presented here. The source terms on the right hand sides of Equations (5) and (6) are introduced below. The term $\rho(x, t)$ on the right hand side of Equation (7) denotes the total space charge density:

$$
\rho=q\left(n_{h}+n_{h t r}-n_{e}-n_{e t r}\right)
$$


As mentioned, the conduction current through the material bulk is due to drift of the injected carriers associated with hopping between shallow traps. To introduce this mechanism, the apparent effective mobilities $\mu_{\mathrm{e}, \mathrm{h}}$ in Equation (4) are defined by the depth of shallow traps $\phi_{\mathrm{e}, \mathrm{h}}$ :

$$
\mu_{e, h}=\mu_{b(e, h)} \exp \left(-\frac{\phi_{e, h}}{k T}\right)
$$

where $\mu_{\mathrm{b}(\mathrm{e}, \mathrm{h})}$ are band mobilities of the respective carriers.

The intensity of trapping process is characterized by trapping coefficients $t_{\mathrm{e}, \mathrm{h}}$ which reflect the probability of capturing of charged species per unit of time. The total trapping rates are quantified as:

$$
T_{e, h}=t_{e, h} n_{e, h}\left(1-\frac{n_{e t r, h t r}}{N_{e t r, h t r}}\right)
$$

where $n_{\mathrm{e}, \mathrm{h}}$ and $n_{\mathrm{etr}, \mathrm{htr}}$ are the number densities of the charge carriers, $N_{\mathrm{etr}, h t r}$ are the total densities of deep traps in the insulating material.

The rates of de-trapping from deep traps, which are considered as potential wells with barrier heights $\phi_{\text {etr,htr }}$, are introduced as:

$$
D T_{e, h}=v n_{e t r, h t r} \exp \left(-\frac{\phi_{e t r, h t r}}{k T}\right) \frac{n_{e t r, h t r}}{N_{e t r, h t r}}
$$

where $v=k T / h$ [7] being the attempt-to-escape frequency and $h$ indicating Planck's constant. The dynamics of filling/releasing traps are described by Equation (6).

In the model, it is assumed that recombination of charges of opposite polarities is mainly between trapped and mobile charges and takes place at trapping sites acting as recombination centers. Probability of recombination between two types of mobile charges is significantly lower $[1,8]$ and can be neglected. In general, the recombination processes lead to the loss in quantity of charged species that are expressed using the rates:

$$
\begin{aligned}
& R_{e h}=r_{e h} n_{e} n_{h} \\
& R_{e t r h}=r_{e t r h} n_{\text {etr }} n_{h} \\
& R_{\text {ehtr }}=r_{\text {ehtr }} n_{e} n_{h t r} \\
& R_{\text {etrhtr }}=r_{\text {etrhtr }} n_{\text {etr }} n_{h t r}
\end{aligned}
$$

Here, $r$ indicates recombination coefficient and the subscripts eh, etrh, ehtr, etrhtr represent recombination between mobile electrons and mobile holes, trapped electrons and mobile holes, mobile electrons and trapped holes, trapped electrons and trapped holes, respectively.

The total rates of generation and losses of mobile and trapped charges in Equations (5) and (6) above can be expressed as:

$$
\begin{aligned}
& S_{e}=-R_{e h}-R_{e h t r}-T_{e}+D T_{e} \\
& S_{h}=-R_{e h}-R_{e t r h}-T_{h}+D T_{h} \\
& S_{e t r}=-R_{\text {etrh }}-R_{\text {etrhtr }}+T_{e}-D T_{e} \\
& S_{\text {htr }}=-R_{\text {ehtr }}-R_{\text {etrhtr }}+T_{h}-D T_{h}
\end{aligned}
$$

Note the signs of the terms in Equations (13) which indicate generation (positive rate) or loss (negative rate) mechanisms. Thus, the mobile charges are lost through trapping and recombination and are gained through de-trapping while the latter is the sink of trapped charges.

Finally, the total measurable time-dependent conduction current density through the sample summarizing the contributions from both types of charge carriers is found as:

$$
J_{\text {cond }}(t)=\frac{1}{L} \int_{0}^{L}\left[j_{h}(x, t)+j_{e}(x, t)\right] \mathrm{d} x
$$


It is well-known that the measured charging current constitutes two components, namely the displacement and conduction currents. As the polarization is modelled by a constant permittivity, the integration of the displacement current over space leads to a term proportional to the time derivative of the voltage, which vanishes exactly for dc applied voltage. Therefore, Equation (14) gives the total current even in the transient phase. Hence, the simulated current density $J_{\text {cond }}(t)$ is used below for comparison with experimentally obtained current density.

The initial and boundary conditions are described as follows. Since a high electric field $(32.5 \mathrm{kV} / \mathrm{mm})$ was applied to the insulation in the experiments, the density of charge carriers in the material corresponding to thermal equilibrium prior to voltage application is assumed to be insignificant as compared to the density of injected carriers. Therefore, the densities $n_{\mathrm{e}, \mathrm{h}}$ and $n_{\mathrm{etr}, \mathrm{htr}}$ are set to zero at $t=0$. Additionally, if charge carriers of certain polarity reach the counter electrode, no extraction barrier for their ejection is specified in the model. In other words, all electrons arriving to the anode and holes arriving to the cathode disappear from the insulation domain. For this, the outward current densities at corresponding electrodes (i.e., $j_{\mathrm{e}}$ at the anode and $j_{\mathrm{h}}$ at the cathode) are determined in accordance to Equation (4).

\subsection{Computer Implementation}

The presented model was utilized for simulations of charge transport in $80 \mu \mathrm{m}$ thick films of LDPE with and without nanofillers under conditions corresponding to the experiments [17]. It was implemented in finite element software COMSOL Multiphysics. Numerical solutions of the equation system (5)-(7) were obtained in one-dimensional computational domain. Suitable application modes provided in the software were selected for solving the continuity equation (5), ordinary differential equation (6), and Poisson's equation (7). The external conduction current density through the sample was calculated by substituting the transport equation (4) into Equation (14). A non-uniform mesh was created with extremely small elements in the vicinity of both electrodes (the smallest element size $0.1 \mu \mathrm{m}$ ), whereas coarser mesh was kept in the middle of the sample. The coupling of different application modes was implemented so that the densities of charge carriers obtained as solutions of Equations (5) and (6) at every time step were updated in Equation (8) for gaining the total space charge density. This was further utilized in Poisson's equation (7) for deriving the electric field distribution.

\section{Results of the Simulations and Discussion}

\subsection{Charge Transport in LDPE without Nanofillers}

The set of model parameters provided an agreement between the computed and measured results is presented in Table 1. The mobility of holes was set close to the values deduced from the measurements [17], while the effective mobility of electrons was approximately one order of magnitude higher than the mobility of holes (as has been found in [20,21]). It should be noted that the potential barrier height at the anode was set to be lower than that at the cathode as the injection of holes from a semiconducting anode was alleviated as compared to the electron injection from a metal cathode [21]. Furthermore, the barrier heights for de-trapping $\sim 1.0 \mathrm{eV}$ were selected in accordance to trap depth level revealed by the results of thermally stimulated currents [22,23] and our calculations [17] based on demarcation energy model. These levels of trap depth are also in agreement with the values used in other numerical model [6]. The trap densities $\sim 10^{21} \mathrm{~m}^{-3}$ were set based on the results obtained in [24]. The recombination coefficients were adopted from [6]. Finally, the trapping coefficients were adjusted to achieve the best fit. The commonly accepted relative permittivity $\varepsilon_{r}=2.3$ was used for LDPE.

The results of the simulations are presented in Figure 1 together with experimental data. Note that the rapid reduction of the measured currents within first 50-70 s particularly prominent at room temperature and $40^{\circ} \mathrm{C}$ in Figure 1, is most likely associated with the decaying displacement current arising due to the application of a step voltage. The conduction current component becomes dominating only at longer instants after voltage application $\left(t \geqslant 10^{2}-10^{3} \mathrm{~s}\right)$ and, hence, simulated and 
experimental currents can be compared only in this stage. As seen in the figure, the current density at room temperature $\left(\sim 22^{\circ} \mathrm{C}\right)$ predicted by the model agrees well with the measured one. At higher temperatures, the computed characteristics are still in line with experimental data but the agreement is getting worse. Broad maxima appear in the simulated currents and the peaks are shifted to a shorter time as the temperature rises.

Table 1. Parameters used in models for fitting the measured conduction currents on LDPE and its nanocomposites at various temperatures. RT stands for room temperature $\left(\sim 22{ }^{\circ} \mathrm{C}\right)$.

\begin{tabular}{|c|c|c|c|c|c|c|}
\hline \multirow{2}{*}{ parameters } & \multicolumn{3}{|c|}{ LDPE } & \multicolumn{3}{|c|}{ LDPE Nanocomposites } \\
\hline & RT & $40^{\circ} \mathrm{C}$ & $60^{\circ} \mathrm{C}$ & RT & $40^{\circ} \mathrm{C}$ & $60{ }^{\circ} \mathrm{C}$ \\
\hline $\begin{array}{l}\text { Effective mobility } \\
\mu_{\mathrm{e}}, \mathrm{m}^{2} \cdot \mathrm{V}^{-1} \cdot \mathrm{s}^{-1} \\
\mu_{\mathrm{h}}, \mathrm{m}^{2} \cdot \mathrm{V}^{-1} \cdot \mathrm{s}^{-1}\end{array}$ & $\begin{array}{l}3.0 \times 10^{-14} \\
2.5 \times 10^{-15}\end{array}$ & $\begin{array}{l}1.5 \times 10^{-13} \\
1.2 \times 10^{-14}\end{array}$ & $\begin{array}{l}5.5 \times 10^{-13} \\
5.0 \times 10^{-14}\end{array}$ & $\begin{array}{l}1.0 \times 10^{-14} \\
2.0 \times 10^{-15}\end{array}$ & $\begin{array}{l}3.0 \times 10^{-14} \\
6.0 \times 10^{-15}\end{array}$ & $\begin{array}{l}7.0 \times 10^{-14} \\
1.4 \times 10^{-14}\end{array}$ \\
\hline $\begin{array}{c}\text { Trapping coefficients } \\
t_{\mathrm{e}}, \mathrm{s}^{-1} \\
t_{\mathrm{h}}, \mathrm{s}^{-1}\end{array}$ & $\begin{array}{l}0.02 \\
0.01\end{array}$ & $\begin{array}{l}0.08 \\
0.03\end{array}$ & $\begin{array}{l}0.25 \\
0.08\end{array}$ & $\begin{array}{l}0.002 \\
0.002\end{array}$ & $\begin{array}{l}0.022 \\
0.022\end{array}$ & $\begin{array}{l}0.13 \\
0.13\end{array}$ \\
\hline $\begin{array}{c}\text { De-trapping barrier height } \\
\phi_{\text {etr }}, \mathrm{eV} \\
\phi_{\mathrm{htr}}, \mathrm{eV} \\
\end{array}$ & $\begin{array}{l}0.93 \\
0.93 \\
\end{array}$ & $\begin{array}{l}0.96 \\
0.96 \\
\end{array}$ & $\begin{array}{l}1.00 \\
1.00 \\
\end{array}$ & & $\begin{array}{l}1.00 \\
1.00\end{array}$ & \\
\hline $\begin{array}{c}\text { Deep trap density } \\
N_{\text {etr }}, \mathrm{m}^{-3} \\
N_{\text {htr }}, \mathrm{m}^{-3}\end{array}$ & & $\begin{array}{l}1.25 \times 10^{21} \\
1.25 \times 10^{21}\end{array}$ & & & $\begin{array}{l}6.25 \times 10^{21} \\
6.25 \times 10^{21}\end{array}$ & \\
\hline $\begin{array}{c}\text { Schottky injection barriers } \\
\phi_{\mathrm{K}}, \mathrm{eV} \\
\phi_{\mathrm{A}}, \mathrm{eV} \\
\end{array}$ & & $\begin{array}{l}1.22 \\
1.16\end{array}$ & & & $\begin{array}{l}1.31 \\
1.26 \\
\end{array}$ & \\
\hline $\begin{array}{c}\text { Recombination coefficients } \\
r_{\text {etrhtr }}, \mathrm{m}^{3} \cdot \mathrm{s}^{-1} \\
r_{\text {etrh }}, \mathrm{m}^{3} \cdot \mathrm{s}^{-1} \\
r_{\mathrm{ehtr}}, \mathrm{m}^{3} \cdot \mathrm{s}^{-1} \\
r_{\mathrm{eh}}, \mathrm{m}^{3} \cdot \mathrm{s}^{-1}\end{array}$ & & $\begin{array}{l}6.4 \times 10^{-22} \\
6.4 \times 10^{-22} \\
6.4 \times 10^{-22} \\
0\end{array}$ & & & $\begin{array}{c}6.4 \times 10^{-22} \\
6.4 \times 10^{-22} \\
6.4 \times 10^{-22} \\
0\end{array}$ & \\
\hline $\begin{array}{l}\text { Relative permittivity } \\
\mathcal{E}_{\mathrm{r}}\end{array}$ & & 2.3 & & & 2.3 & \\
\hline
\end{tabular}

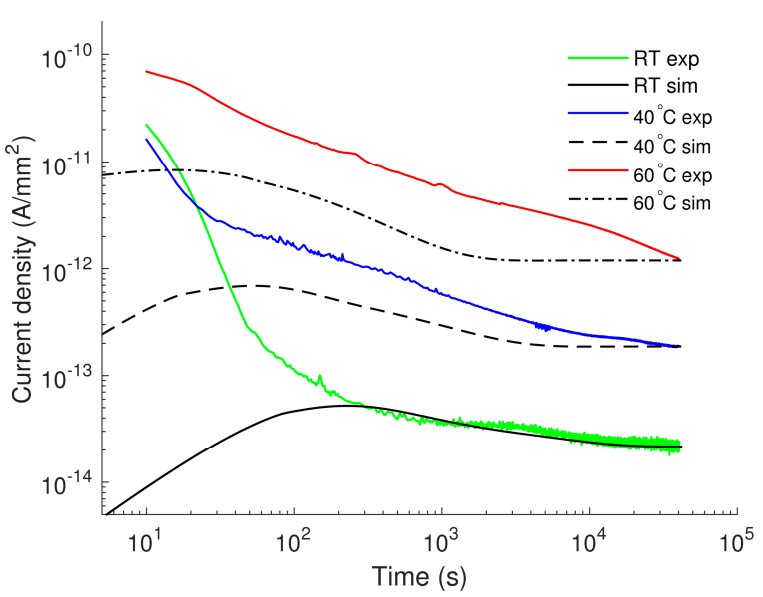

Figure 1. Simulated (referred as "sim" in the legend) and experimental (exp) current densities on LDPE at various temperatures.

Distributions of space charges in the material bulk obtained from the simulations at room temperature are illustrated in Figure 2. As seen, the positive carriers dominated over the negative ones throughout the simulated time interval. The dynamics of the space charges in the material can be 
characterized by their transit times determined as the time duration required for charges traversing through the insulation bulk. The arrival of holes to the cathode and electrons to the anode can be traced by using the profiles of mobile charge density shown in Figure 3. Based on that, the transit time $\sim 100 \mathrm{~s}$ for electrons and $\sim 1000 \mathrm{~s}$ for holes can be identified that is consistent with the higher (almost one order of magnitude) mobility of electrons as indicated in Table 1. Additionally, the obtained transit times of charge carriers are very close to values calculated as $t_{\mathrm{tr}}=L^{2} /\left(\mu V_{0}\right)$ assuming insignificant accumulation of space charges in the bulk. As it is observed in Figure 2c, the latter is true for the time shorter than the transit time.


Figure 2. Computed charge density distributions in LDPE at room temperature: (a) mobile charges; (b) trapped charges; and (c) total space charges. Positions of the anode and cathode are indicated by letters $\mathrm{A}$ and $\mathrm{K}$, respectively.

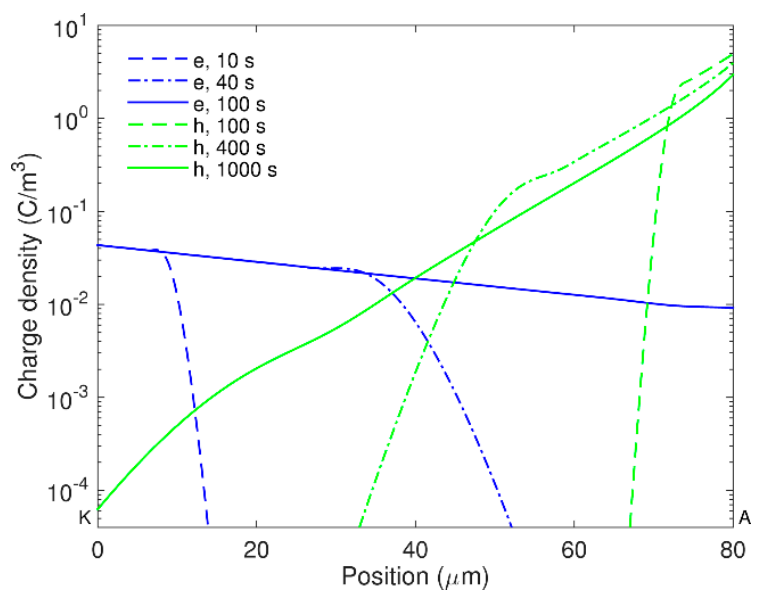

Figure 3. Density profiles of mobile electrons (e) and holes (h) in LDPE at room temperature computed at indicated time.

During the transport of injected holes towards the cathode, their density reduces remarkably due to the trapping process and most of the mobile carriers concentrate within a thin layer $(5-10 \mu \mathrm{m})$ at the vicinity of the anode (Figure 2a). The accumulation of mobile positive carriers in the bulk takes place mostly within first $100 \mathrm{~s}$; thereafter, a reduction in their density can be observed. As seen in Figure $2 b$, immobile positive charges are gradually built up in the vicinity of the anode within the time interval $10^{2}-10^{3} \mathrm{~s}$ and its density becomes much higher after $10^{3} \mathrm{~s}$. Thus, the immobile charges are strongly dominating over the mobile ones in the material bulk. The dynamics of positive charge accumulation are controlled by charge injection before $10^{2} \mathrm{~s}$ and by charge trapping after $10^{3} \mathrm{~s}$, while a transition process takes place in the time interval $10^{2}-10^{3} \mathrm{~s}$. A similar tendency is also observed for the negative charges. In particular, the onset of negative charge accumulation in trapping sites close to the cathode is observed at $\sim 10^{3} \mathrm{~s}$, which results in a considerable amount of trapped electrons in the bulk after $10^{4} \mathrm{~s}$. The variation in the density of the mobile charges leads to the corresponding changes in the simulated conduction current and, hence, a broad maximum appears at time 200-300 s. It should be 
emphasized that the contribution of mobile electrons to the conduction current cannot be neglected in spite of their remarkably lower density as compared to that of mobile holes. This is because of the higher mobility of electrons than that of holes.

The distribution and evolution of space charges in LDPE at elevated temperatures are not shown here as the main features presented above are preserved. However, one should mention three distinctions, namely, (a) higher levels of charge densities owing to a larger amount of charges injected at elevated temperatures; (b) faster charge transport processes as charge carriers become more mobile with increasing temperature; and (c) the saturation in the computed conduction currents (see Figure 1) observed at $\sim 10^{4} \mathrm{~s}$ for $40^{\circ} \mathrm{C}$ and at $2 \times 10^{3} \mathrm{~s}$ for $60^{\circ} \mathrm{C}$. The last feature is not observed within the considered time interval (up to $4 \times 10^{4} \mathrm{~s}$ ) in the simulation at room temperature.

\subsection{Charge Transport in LDPE Nanocomposites}

In this section, charge transport in LDPE filled with $3 \mathrm{wt} \%$ of $\mathrm{Al}_{2} \mathrm{O}_{3}$ and $\mathrm{MgO}$ nanoparticles is studied. As it is indicated [17], the $\mathrm{Al}_{2} \mathrm{O}_{3}$ particles have spherical shape with an average diameter of $40 \mathrm{~nm}$, whereas the $\mathrm{MgO}$ nanoparticles are in rounded hexagonal shape with an average size of $66 \mathrm{~nm}$ and a thickness of 10-20 nm. Since the respective volume fractions of the nanofillers are low $(0.7-0.8 \mathrm{vol} \%)$, the model used in Section 3.1 can also be employed for heterogeneous materials taking into account the effective medium approximations of properties of the composites. Since the experimental results are very close for both nanocomposites under consideration [17], current densities obtained on LDPE $/ \mathrm{Al}_{2} \mathrm{O}_{3} 3 \mathrm{wt} \%$ are employed for comparison with the simulated ones.

\subsubsection{Model Parameterization}

As it is shown in the first part of the work [17], the measured dc conductivity is substantially lower for LDPE filled with $3 \mathrm{wt} \%$ of nanofillers as compared to the unfilled LDPE that is believed to be associated with the weaken charge transport in the nanofilled materials. By recalling the well-known expression for dc conductivity $\sigma=q \sum n_{\mathrm{i}} \mu_{\mathrm{i}}$, (where $n_{\mathrm{i}}$ and $\mu_{\mathrm{i}}$ respectively stand for the density and mobility of $i^{\text {th }}$ type of charge carriers participating in the transport), the reduction in dc conductivity of nanocomposites can be quantitatively related to the decrease in the density of charge carriers and/or the effective mobility.

Despite the density of mobile charge carriers cannot be monitored separately from trapped carriers in space charge measurements, the concentration of mobile charges is anticipated to be lower in nanocomposites. Significant suppression of space charge accumulation in PE nanocomposites observed in various works $[13,18,19]$ has been interpreted by presence of deep traps. Takada et al. [12] explained the origin of the deep traps as potential wells induced at the surface of nanoparticles. The depth of potential wells increases strongly with the applied electric field and the dielectric permittivity of the fillers. Thus for LDPE/MgO nanocomposite, the trap depth may be 1-5 eV with the highest level corresponding to the applied field strength of $\sim 200 \mathrm{kV} / \mathrm{mm}$. Further, the trap depth of $\sim 2 \mathrm{eV}$ has been detected in LDPE/MgO nanocomposite by analyzing results of thermally stimulated currents and the origin of these deep traps have been explained by the effect of nanofillers [25]. Based on these findings, the trap depth should be set higher than that for unfilled LDPE. In addition, increased concentrations of traps has been found in nanofilled PE [24]. Such modifications are expected to enhance capturing of charge carriers injected from the electrodes that may result in thinner layers of homocharges in the vicinity of the electrodes as compared to the case of pure material. These, in turn, may reduce the field strength at the interfaces and so decrease the injected currents [24]. This phenomenon can be identified as a screening effect produced by accumulated homocharges. According to the analysis [13], the screening effect yields a higher barrier height for charge injection at electrodes in case of PE-based nanocomposites as compared to the unfilled counterpart. Considering these modifications in material properties brought about by nanofillers, the barrier heights for charge injection at both electrodes were increased by up to $0.1 \mathrm{eV}$ and the density of deep traps rose in five times for the nanocomposites as compared to the reference LDPE (see Table 1). As regards the mobility of charge carriers in 
nanocomposites, reduced values have been found experimentally [17], which can be elucidated by the alternation in the amorphous region of PE by nanoparticles [26].

According to results of dielectric spectroscopy measurements [27] conducted on LDPE and $\mathrm{LDPE} / \mathrm{Al}_{2} \mathrm{O}_{3} 3 \mathrm{wt} \%$ nanocomposite in frequency range $10^{-4}-10^{3} \mathrm{~Hz}$ and at three temperatures considered in the model, the relative permittivity was slightly higher (maximum $5 \%$ ) for the nanocomposite than for reference LDPE. Additionally, the frequency dependencies of the relative permittivity were weak for both materials. The relative permittivity of LDPE nanocomposite was therefore set to 2.3 as for the unfilled LDPE.

\subsubsection{Results}

The experimental and computed currents in the nanomaterial are compared in Figure 4. Unlike the case of pure LDPE, the conduction current densities predicted by the model show good agreement with the measured ones at all three considered temperatures. At each temperature, the localized peak in the simulated conduction current appears later for the nanocomposite as compared to reference LDPE (Figure 1) due to the lower mobility of charge carriers in nanofilled material.



Figure 4. Current densities obtained from simulations (sim) and experiments (exp) on $\mathrm{LDPE} / \mathrm{Al}_{2} \mathrm{O}_{3}$ nanocomposite at various temperatures.

Distributions of charge densities in the bulk of the nanocomposite are shown in Figure 5 for room temperature. Similarly to the reference LDPE, positive charge carriers are dominating in the material and they are mainly concentrated in a thin layer at the vicinity of the anode. As expected, the amount of charges accumulated in the bulk of LDPE nanocomposite is significantly smaller as compared to that in the reference material. Thus, the maximum density of mobile carriers is almost 50 times lower (compare Figures $2 a$ and $5 a$ ) while the total space charge density is less than $5 \mathrm{C} / \mathrm{m}^{3}$ in most part of the nanocomposite (positions $0-70 \mu \mathrm{m}$ ) and its maximum at the anode is below $9 \mathrm{C} / \mathrm{m}^{3}$, Figure $5 \mathrm{c}$. In the unfilled LDPE, the space charges with density exceeding $5 \mathrm{C} / \mathrm{m}^{3}$ propagate deeply into the bulk and its maximum is at least six times higher $\left(\sim 55 \mathrm{C} / \mathrm{m}^{3}\right)$, see Figure $2 \mathrm{c}$. As a result, the electric field is strongly enhanced inside the reference material, but this is not the case for the nanocomposite. As it is seen in Figure 6, the distortion in electric field distribution in the nanofilled material is negligible at $1 \mathrm{~h}$ after voltage application and only small $(\sim 7 \%)$ field enhancement is observed at the vicinity of the cathode at $4 \times 10^{4} \mathrm{~s}$. On the contrary, an appreciable enhancement $(\sim 25 \%)$ can be noticed in the middle of the sample of the unfilled LDPE at $4 \times 10^{4} \mathrm{~s}$.

The quantity of accumulated positive space charges (dominating carriers) calculated as:

$$
Q(t)=\int_{0}^{L} q\left[\mathrm{n}_{h}(x, t)+\mathrm{n}_{h t r}(x, t)\right] \mathrm{d} x
$$


is presented as a function of time in Figure 7 for all three temperatures. As seen, the amount of charge steadily rises with time and eventually reaches a saturation level $\sim 10^{-3} \mathrm{C} / \mathrm{m}^{2}$. The charge magnitudes are lower in the LDPE nanocomposite for all studied temperatures and the differences are more than one order of magnitude in the short time interval, whereas they become smaller at longer time. For simulations at elevated temperatures and time exceeding $10^{4} \mathrm{~s}$, the total positive charges are comparable in both materials.
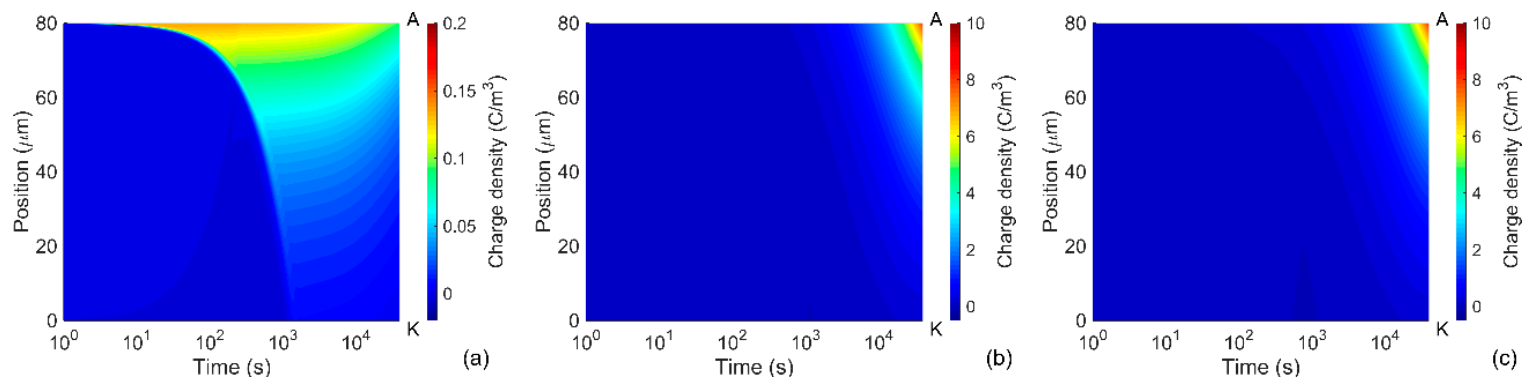

Figure 5. Distributions of charge densities in LDPE nanocomposite obtained from simulations at room temperature: (a) mobile charges; (b) trapped charges; and (c) total space charges. Positions of the anode and cathode are indicated by letters $\mathrm{A}$ and $\mathrm{K}$, respectively.



Figure 6. Electric field distributions in LDPE without (Ref) and with (NC) nanofillers at room temperature.



Figure 7. Amount of positive charges accumulated in the bulk of LDPE (Ref) and LDPE nanocomposite (NC). 


\subsection{Influence of Different Physical Processes on Charge Transport}

As discussed above, the weakening in charge transport in nanofilled LDPE as compared to the unfilled one can be attributed to the reduced charge injection at electrode-insulation interfaces, to the decreased charge carrier mobility, to the increased probability of charge capturing in and the decrease of charge release from deep traps. However, it is unclear which process among the above-mentioned mainly contributes to the lowering of the conduction in LDPE nanocomposite. In other words, what behavior of insulation is changed most noticeable due to the addition of nanofillers into LDPE?

To address this question, we assume that only one type of parameters incorporated in the model for pure LDPE and associated with a certain physical process is modified at a time to the values used in the model of LDPE nanocomposite (Table 1), while all other parameters are kept unchanged. Thus, four scenarios are considered as described in Table 2 and the obtained results (conduction currents at temperature $40^{\circ} \mathrm{C}$ ) are illustrated in Figure 8, where the simulated currents in LDPE and its nanocomposite are also shown for comparison. As can be seen, the conduction current drops significantly down to the level close to that in the nanocomposite while increasing the injection barriers alone and less pronounced decline is observed in three other situations. The effect of charge mobility on the conduction current is almost the same in the studied time interval, whereas the influence of trap energy $\left(\varphi_{\text {tr }}\right)$ and trap density $\left(N_{\text {tr }}\right)$ is remarkable only at times exceeding $10^{4} \mathrm{~s}$. Based on the results of the analysis, we found that the decrease in charge injection at the electrodes mainly accounts for the weakening of conduction in LDPE nanocomposite and so for the suppression of space charge build-up in the bulk.

Table 2. Scenarios for simulations with varying parameters. Model parameters of each scenario are the same as for simulating charge transport in LDPE, except for those provided in the right column. Charge mobilities (in $\mathrm{m}^{2} \cdot \mathrm{V}^{-1} \cdot \mathrm{s}^{-1}$ ) are listed in order of increasing temperature (RT; $40{ }^{\circ} \mathrm{C} ; 60{ }^{\circ} \mathrm{C}$ ).

\begin{tabular}{|c|c|c|c|}
\hline Scenario & Description & Model parameters & Modified parameters \\
\hline$\# 1$ & $\begin{array}{l}\text { Reduction of charge } \\
\text { injection at electrodes }\end{array}$ & $\begin{array}{l}\text { Charge injection barrier heights as for the } \\
\text { nanocomposite, all other parameters as for LDPE }\end{array}$ & $\begin{array}{l}\phi_{\mathrm{K}}=1.31 \mathrm{eV} \\
\phi_{\mathrm{A}}=1.26 \mathrm{eV}\end{array}$ \\
\hline \#3 & $\begin{array}{l}\text { Reduction of charge } \\
\text { carrier mobility }\end{array}$ & $\begin{array}{l}\text { Mobility of electrons and holes as for the } \\
\text { nanocomposite, all other parameters as for LDPE }\end{array}$ & $\begin{array}{c}\mu_{\mathrm{e}}=1 \times 10^{-14} ; 3 \times 10^{-14} ; 7 \times 10^{-14} \\
\mu_{\mathrm{h}}=2 \times 10^{-15} ; 6 \times 10^{-15} ; 1.4 \times 10^{-14}\end{array}$ \\
\hline
\end{tabular}

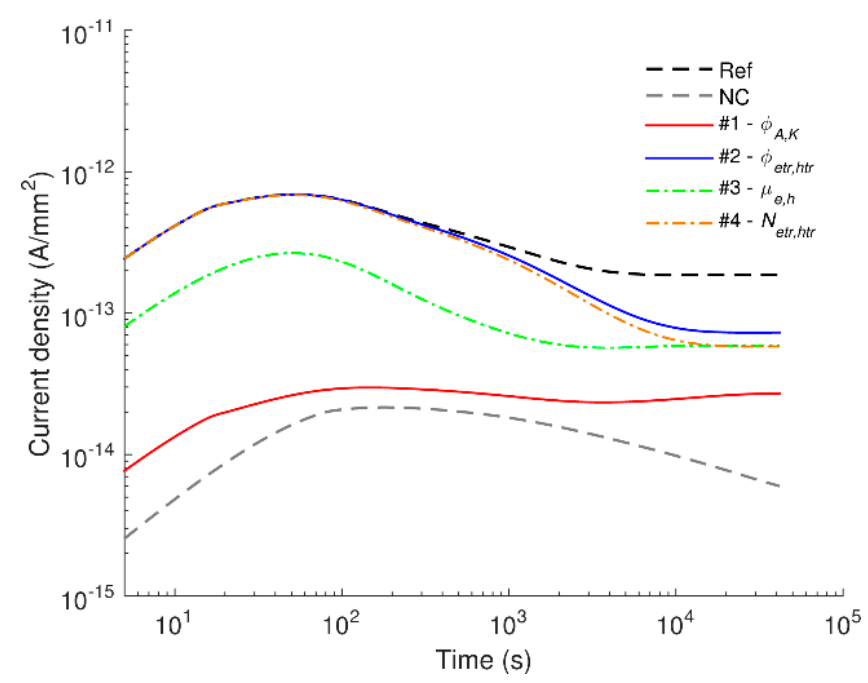

Figure 8. Simulated conduction currents at $40{ }^{\circ} \mathrm{C}$ obtained by varying model parameters. Four scenarios are considered as shown in Table 2 . The parameters being changed are indicated in the legend. 
The contribution of charge recombination to the conduction current is examined by considering charge transport models with and without accounting for this particular process. The simulated conduction currents in both materials are compared in Figure 9. As it is found, charge recombination is essential in pure material and in the nanocomposite at $60^{\circ} \mathrm{C}$. Neglecting this process yields a rapid rise of the simulated currents, especially at elevated temperatures. The marked increase in the conduction currents obtained in the model without recombination is due to the excess of mobile charges in the bulk, which would be neutralized if recombination is included. In this context, it is interesting to observe that such neutralization is not of importance for the nanofilled material at room temperature and at $40{ }^{\circ} \mathrm{C}$. The differences in the simulated outcomes for LDPE with and without nanoparticles can be attributed to the strong distinctions in the amount of charge carriers generated in these materials. The obtained results also indicate that charge recombination cannot be underestimated in the charge transport model at elevated temperatures, even though its contribution is minor at room temperature.


Figure 9. Simulated conduction currents obtained from models with (solid curves) and without (dashed curves) charge recombination.

\subsection{Discussion}

As mentioned above, broad maxima are observed in the simulated time variations of the current densities and the time $t_{\mathrm{p}}$ corresponding to the current peaks is temperature-dependent (see Figures 1 and 4 ). These localized peaks are not exhibited in our experimental results [17]. Indeed, localized peaks are often detected in time-domain currents measured on oxidized LDPE [28] rather than on the non-oxidized counterpart [29]. Their appearance has been explained by the high concentration of carbonyl groups $(-\mathrm{C}=\mathrm{O})$ in the former material as compared to the latter. The carbonyl groups give rise to the hopping transport of mobile charge carriers in the bulk that eventually increases the conduction current [28]. Current maxima are therefore observed as a consequence of the build-up of significant mobile charges in the bulk [30]. In contrast, lower conduction currents are detected for the non-oxidized PE and the peaks are most probably hidden by the displacement current. The latter arises in transient processes activated by the voltage application due to orientation of polar groups existing in PE. In LDPE samples used in this investigation, the presence of antioxidant is anticipated to suppress the formation of carbonyl groups that explains the absence of the current maxima in the measured charging currents. Nevertheless, the current maxima in the simulated characteristics are of interest. According to the analysis by Many and Rakavy [30] for a single-carrier model in trap-free materials, the peak of transient current corresponds to the arrival of charges at the counter electrode. The peak time $t_{\mathrm{p}}$ can be found as $t_{\mathrm{p}}=0.787 \times t_{\mathrm{tr}}$, where $t_{\mathrm{tr}}=L^{2} /\left(\mu V_{0}\right)$. Unlike the case of trap-free materials, different features are noted in the bipolar charge transport model for materials with traps. As charge trapping strongly reduces the density of mobile carriers, their total density and the current density achieve maxima well before the arrival of the dominating charge carriers at the 
counter electrode. Thus, in a correlation between $t_{\mathrm{p}}$ and $t_{\mathrm{tr}}$ established by using results obtained in Sections 3.1 and 3.2.2 the multiplication factor should be much lower than 0.787.

Another noteworthy feature is that the accumulation of trapped charges in the unfilled LDPE at elevated temperatures becomes saturated after certain time, e.g., at $2 \times 10^{3} \mathrm{~s}$ at $60^{\circ} \mathrm{C}$. As the trapped charges constitute the main part of the space charges, the same tendency is observed for the latter, yielding unchanged electric field distribution in the insulation bulk afterward. This eventually causes the steady state of simulated current density as seen in Figure 1. In order to avoid the early saturation in the simulated external current density, the trapping coefficients have been adjusted as increasing with temperature, which can be interpreted as the increased probability of charge trapping due to the presence of an increased amount of charges generated at higher temperatures. However, we realized that the steady state in the current density is persistent for simulations at elevated temperatures and it is unavoidable for the described model of charge transport. In fact, the saturation in the simulated trapped charges has been noted in [5] at $9 \times 10^{3} \mathrm{~s}$ and the saturated conduction currents are clearly illustrated in [14]. In both cases, the simulations of charge transport were implemented for the conditions of ambient temperature. Note that at room temperature, the steady state in the simulated characteristics is not exhibited within the considered time range in the present study; it only arises at elevated temperatures. The effect of temperature on the saturation of the simulated characteristics could be attributed to the fact that the injected currents described by Schottky's law, Equations (1) and (2), may not fully reflect the physical processes at the electrodes. According to Schottky's law, the amount of injected mobile carriers at the electrodes increases substantially with temperature and, hence, the traps in the insulation bulk can be filled more easily at higher temperatures, yielding the premature saturation in the density of trapped charges and so for the simulated conduction currents.

The applicability of Schottky's mechanism for charge injection at electrode-insulation interfaces is in fact questionable [31]. First of all, the distance $x_{\max }$ from the electrode corresponding to the maximum of potential barrier is too long so that an electron may be thermalized by collisions before reaching the barrier [31]. Secondly, the barrier height for injection used in simulations ( 1.1-1.3 eV) is much smaller than that at metal-PE interfaces obtained by using density functional theory (DFT) calculations, e.g., [32]. Taylor and Lewis [33] analyzed currents measured on thin films of polyethylene terephthalate (PET) and PE exposed to a wide range of applied electric field at various temperatures and proposed an alternative to Schottky's mechanism where a general form followed Equation (16) instead of the coulombic form by Equation (17) of the potential barrier at electrodes is utilized:

$$
\begin{gathered}
\phi_{G}(x)=-\frac{K q}{(a x)^{n}} \\
\phi_{C}(x)=-\frac{q^{2}}{16 \pi \varepsilon x}
\end{gathered}
$$

In Equation (16), $K, a$, and $n$ are positive constants, $K$ accounts for contributions of the charge $q$ and material permittivity $\varepsilon$ presented in the coulombic form of Equation (17). In Equations (16) and (17), $x$ is the distance from the electrode. The widely used Schottky injection law with the coulombic form of the potential barrier is a special case of the general form when the exponent $n$ equals unity. For PET and PE, it has been found that the constant $n$ is much lower than unity. The departure from the image-law potential barrier according to Equation (17) has been explained by space charge build-up at the interfaces and in the insulation bulk as well [33]. Additionally, zero-field activation energies derived for PET and PE were respectively 2.58 and $2.14 \mathrm{eV}$, which were interpreted as the potential barriers of the general law [33]. These values are closer to the results of DFT calculations obtained recently [32] as compared to the commonly used ones in the simulations. It should be mentioned that transient processes due to charge trapping, de-trapping, and recombination in the bulk have not been considered in these analyses [33] and, hence, the proposed approach should be reconsidered by taking into account the bulk processes. This may provide better explanation of experimental data obtained for the reference LDPE at elevated temperatures. 


\section{Conclusions}

Charge transport in LDPE and its nanocomposites at different temperatures has been studied by numerical simulations. By achieving a good agreement between simulated and measured conduction currents, we reveal quantitative changes in various physical processes taking place in the insulating materials caused by the presence of nanofillers. In particular, the weakening charge transport in the nanodielectrics as compared to the unfilled LDPE is associated with the increased barrier heights for charge injection at electrodes, the reduced charge mobility, and the increased trap energy and trap density. Simulated results also demonstrate that space charge accumulation and electric field enhancement are less noticeable in LDPE nanocomposites than in the unfilled counterpart. The reduced conduction currents and the suppression of space charge accumulation in nanofilled LDPE are most likely governed by the modification of the barrier heights for charge injection at the dielectric-electrode interfaces. Furthermore, the application of Schottky's mechanism for describing charge injection at electrodes in the simulation is still questionable as it does not fully explain the thermally activated behavior of the conduction currents obtained experimentally.

Acknowledgments: The financial support provided by Chalmers Area of Advance in Energy and Swedish Foundation for Strategic Research is gratefully acknowledged.

Author Contributions: Anh T. Hoang implemented the computer model, performed simulations and analyzed the results. Yuriy V. Serdyuk contributed to the development of the computer model and interpretation of the results. Stanislaw M. Gubanski provided support for the analysis of the data. All authors discussed the results and contributed to the preparation of the manuscript.

Conflicts of Interest: The authors declare no conflict of interest.

\section{References}

1. Alison, J.M.; Hill, R.M. A model for bipolar charge transport, trapping and recombination in degassed crosslinked polyethene. J. Phys. D Appl. Phys. 1994, 27, 1291-1299. [CrossRef]

2. Li, Y.; Takada, T. Experimental observation of charge transport and injection in XLPE at polarity reversal. J. Phys. D Appl. Phys. 1992, 25, 704-716. [CrossRef]

3. Fukuma, M.; Nagao, M.; Kosaki, M. Computer Analysis on Transient Space Charge Distribution in Polymer. In Proceedings of the 4th International Conference on Properties and Applications of Dielectric Materials, Brisbane, Queensland, Australia, 3-8 July 1994.

4. Kaneko, K.; Mizutani, T.; Suzuoki, Y. Computer simulation on formation of space charge packets in XLPE films. IEEE Trans. Dielectr. Electr. Insul. 1999, 6, 152-158. [CrossRef]

5. Le Roy, S.; Segur, P.; Teyssedre, G.; Laurent, C. Description of bipolar charge transport in polyethylene using a fluid model with a constant mobility: Model prediction. J. Phys. D Appl. Phys. 2004, 37, 298-305. [CrossRef]

6. Le Roy, S.; Teyssedre, G.; Laurent, C.; Montanari, G.C.; Palmieri, F. Description of charge transport in polyethylene using a fluid model with a constant mobility: Fitting model and experiments. J. Phys. D Appl. Phys. 2006, 39, 1427-1436. [CrossRef]

7. Boufayed, F.; Teyssèdre, G.; Laurent, C.; Roy, S.L.; Dissado, L.A.; Ségur, P.; Montanari, G.C. Models of bipolar charge transport in polyethylene. J. Appl. Phys. 2006, 100, 104105. [CrossRef]

8. Serdyuk, Y.V.; Gubanski, S.M.; Mohammad, A.S. Computer Simulations of Space Charge Dynamics and Electric Fields in HVDC Cable Insulation. In Proceedings of the 15th International Symposium on High Voltage Engineering, Ljubljana, Slovenia, 27-31 August 2007; p. T8-405.

9. Le Roy, S.; Vu, T.T.N.; Laurent, C.; Teyssedre, G. Modelling charge generation and transport in solid organic dielectrics under DC stress. In Proceedings of the European Seminar on Materials for HVDC Cables and Accessories: Performance, Modeling, Testing, Qualification (Jicable HVDC'13), Perpignan, France, 18-20 November 2013; p. 4.1.

10. Taleb, M.; Teyssedre, G.; Le Roy, S.; Laurent, C. Modeling of charge injection and extraction in a metal/polymer interface through an exponential distribution of surface states. IEEE Trans. Dielectr. Electr. Insul. 2013, 20, 311-320. [CrossRef] 
11. Baudoin, F.; Laurent, C.; Teyssedre, G.; Le Roy, S. Charge packets modeling in polyethylene. Appl. Phys. Lett. 2014, 104, 152901. [CrossRef]

12. Takada, T.; Hayase, Y.; Tanaka, Y.; Okamoto, T. Space charge trapping in electrical potential well caused by permanent and induced dipoles for LDPE/MgO nanocomposite. IEEE Trans. Dielectr. Electr. Insul. 2008, 15, 152-160. [CrossRef]

13. Zhang, L.; Zhou, Y.; Tian, J.; Sha, Y.; Zhang, Y.; Wu, H.; Wang, Y. Experiment and simulation of space charge suppression in LDPE/MgO nanocomposite under external DC electric field. J. Electrostat. 2014, 72, 252-260. [CrossRef]

14. Min, D.; Wang, W.; Li, S. Numerical analysis of space charge accumulation and conduction properties in LDPE nanodielectrics. IEEE Trans. Dielectr. Electr. Insul. 2015, 22, 1483-1491. [CrossRef]

15. Fleming, R.J.; Ammala, A.; Lang, S.B.; Casey, P.S. Conductivity and space charge in LDPE containing nanoand micro-sized ZnO particles. IEEE Trans. Dielectr. Electr. Insul. 2008, 15, 118-126. [CrossRef]

16. Murakami, Y.; Nemoto, M.; Okuzumi, S.; Masuda, S.; Nagao, M.; Hozumi, N.; Sekiguchi, Y. DC conduction and electrical breakdown of MgO/LDPE nanocomposite. IEEE Trans. Dielectr. Electr. Insul. 2008, 15, 33-39. [CrossRef]

17. Hoang, A.T.; Pallon, L.; Liu, D.; Serdyuk, Y.V.; Gubanski, S.M.; Gedde, U.W. Charge transport in LDPE nanocomposites Part I-Experimental approach. Polymers 2016, 8, 87. [CrossRef]

18. Hayase, Y.; Aoyama, H.; Tanaka, Y.; Takada, T.; Murata, Y. Space Charge Formation in LDPE/MgO Nano-Composite Thin Film under Ultra-High DC Electric Stress. In Proceedings of the 8th International Conference on Properties and Applications of Dielectric Materials, Bali, Indonesia, 26-30 June 2006; pp. 159-162.

19. Kanegae, E.; Ohki, Y.; Tanaka, T.; Sekiguchi, Y.; Murata, Y.; Reddy, C.C. Space Charge Behavior in Multi-Layered Dielectrics with LDPE and LDPE/MgO Nanocomposites. In Proceedings of the 10th IEEE International Conference on Solid Dielectrics (ICSD), Potsdam, Germany, 04-09 July 2010; pp. 1-4.

20. Toomer, R.; Lewis, T.J. Charge trapping in corona-charge polyethylene films. J. Phys. D Appl. Phys. 1980, 13, 1343-1356. [CrossRef]

21. Chen, G.; Tay, T.Y.G.; Davies, A.E.; Tanaka, Y.; Takada, T. Electrodes and charge injection in low-density polyethylene using the pulsed electroacoustic technique. IEEE Trans. Dielectr. Electr. Insul. 2001, 8, 867-873. [CrossRef]

22. Fischer, P.; Röhl, P. Thermally stimulated and isothermal depolarization currents in low-density polyethylene. J. Polym. Sci. 1976, 14, 531-542. [CrossRef]

23. Mizutani, T.; Suzuoki, Y.; Ieda, M. Thermally stimulated currents in polyethylene and ethylene-vinyl-acetate copolymers. J. Appl. Phys. 1977, 48, 2408-2413. [CrossRef]

24. Tian, F.; Lei, Q.; Wang, X.; Wang, Y. Effect of deep trapping states on space charge suppression in polyethylene/ZnO nanocomposite. Appl. Phys. Lett. 2011, 99, 142903. [CrossRef]

25. Ishimoto, K.; Tanaka, T.; Ohki, Y.; Sekiguchi, Y.; Murata, Y. Thermally stimulated current in low-density polyethylene/MgO nanocomposite. On the mechanism of its superior dielectric properties. Electr. Eng. Jpn. 2011, 176, 1-7. [CrossRef]

26. Lewis, T.J. Charge transport in polyethylene nano dielectrics. IEEE Trans. Dielectr. Electr. Insul. 2014, 21, 497-502. [CrossRef]

27. Hoang, A.T.; Serdyuk, Y.V.; Gubanski, S.M. Dielectric spectroscopy measurements in frequency range $10^{-4}-10^{3} \mathrm{~Hz}$ and at various temperatures (ambient temperature, 40 and $60{ }^{\circ} \mathrm{C}$ ) show similarity in the relative permittivities of the reference $\mathrm{LDPE}$ and $\mathrm{LDPE} / \mathrm{Al}_{2} \mathrm{O}_{3} 3 \mathrm{wt} \%$ nanocomposite as well as their weak dependencies on frequency, 2015.

28. Fischer, P.; Röhl, P. Transient currents in oxidized low-density polyethylene. In Mehrphasige Polymersysteme; Fischer, E.W., Horst Müller, F., Kausch, H.H., Eds.; Steinkopff: Heidelberg, Germany, 1977; Volume 62, pp. 149-153.

29. Pelissou, S.; St-Onge, H.; Wertheimer, M.R. Electrical conduction of polyethylene below and above its melting point. IEEE Trans. Electr. Insul. 1988, 23, 325-333. [CrossRef]

30. Many, A.; Rakavy, G. Theory of transient space-charge-limited currents in solids in the presence of trapping. Phys. Rev. 1962, 126, 1980-1988. [CrossRef]

31. Teyssedre, G.; Laurent, C. Charge transport modeling in insulating polymers: From molecular to macroscopic scale. IEEE Trans. Dielectr. Electr. Insul. 2005, 12, 857-875. [CrossRef] 
32. Chen, L.; Tran, D.H.; Quintero, Y.C.; Ramprasad, R. Charge injection barriers at metal/polyethylene interfaces. J. Mater. Sci. 2015, 51, 506-512. [CrossRef]

33. Taylor, D.M.; Lewis, T.J. Electrical conduction in polyethylene terephthalate and polyethylene films. J. Phys. D Appl. Phys. 1971, 4, 1346-1357. [CrossRef]

(c) 2016 by the authors; licensee MDPI, Basel, Switzerland. This article is an open access article distributed under the terms and conditions of the Creative Commons by Attribution (CC-BY) license (http:/ / creativecommons.org/licenses/by/4.0/). 